Research Report

\title{
Performance of In-Line Microfluidic Mixers in Laminar Flow for High-Throughput Flow Cytometry
}

BioTechniques 33:220-226 (July 2002)

\author{
W. Coyt Jackson, T.A. Bennett, \\ B.S. Edwards, E. Prossnitz, \\ G.P. Lopez, and L.A. Sklar \\ University of New Mexico \\ College of Engineering, \\ Albuquerque, NM, USA
}

\section{INTRODUCTION}

In the burgeoning biotechnology industry, accurate analysis of the interactions between cells and compounds is essential to drug discovery. The current cost of compounds renders sample size paramount and favors microfluidic systems and submicroliter volumes. When governed by laminar flow alone, such systems are inherently subject to slow mixing (1,3). Alternate devices where microfluidic mixing takes place have not been systematically incorporated with high-throughput flow cytometry [Reference 6; www.gesim.de/mfscomp.htm (GeSiM - Microsystems/ Components) and www.aps.org/meet/ DFD00/baps/abs/S1270.html\#SJE.009 (53rd Annual Meeting of the Division of Fluid Dynamics of the American Physical Society, November 19-21, 2000; Washington, D.C)]. Commonly used microfluidic mixers are diffusionenhanced or highly complex and require periods of at least seconds in which to thoroughly mix $(4,8)$. In the micrometer size range, small Reynolds numbers govern the delivery of aqueous samples. As fluid transport systems get progressively smaller, viscous forces dominate inertial forces, thus rendering turbulence nonexistent. Innovative mixing platforms incorporating soft lithography (10) microsystems have also been described.

We previously described the performance of a Y combined with a peristaltic pump and liquid samples separated by air bubbles $(5,7)$. This sample handler delivers compound samples from a 96-well plate and a continuous particle-containing stream. The two fluid streams enter through two bifurca- tions of a Y fitting and exit at a common central outlet that leads to the analysis laser. The outlet stream is then connected to a flow cytometer via an adapter constructed in-house and connected to a length of tubing that we dubbed the "mixing window". Conventionally the samples remain in laminar flow and mix slowly via diffusion. Mixing in this system apparently results from the combination of the Y, bubbles, and pulsatile fluid flow. When the cell or bead sample is driven by a syringe and the compounds by a peristaltic pump (Figure 1A), the peristalsis is dampened and the degree of pulsatile mixing is reduced. To adequately mix these two streams, a substantial degree of perturbation is required, which we show here to be provided by an in-line micromixer detected in real time for cytometric analysis.

To qualitatively analyze our mixing device, we used high-throughput flow cytometry combined with two separate fluorescent reactions. First, the degree of mixing was determined through use of an avidin bead-based assay in which an avidin-bound FITC-biotin was quenched on the bead and free biotin was mixed to produce fluorescence unquenching. Second, we used a cell-based assay where a formyl peptide ligand was combined with a receptor-transfected cell line loaded with the calcium-sensing Fluo-4 dye to induce a receptor-ligand-driven calcium response to increase fluorescence. In both of these high-throughput assays, efficient mixing was observed. This simple approach to mixing in com bination with high-throughput flow cytometry represents an important addition to a rapid and accurate analysis tool for a wide variety of assays. 


\section{MATERIALS AND METHODS}

\section{In-Line Mixer Materials}

Submillimeter wire $[120 \mu \mathrm{m}, 0.005$ in o.d. (Small Parts, Miami Lakes, FL, USA)] was divided into 500- $\mu \mathrm{m}$ lengths to comprise the initial in-line microfluidic mixing wires (Figure 1B). A magnetic stirring mechanism (Scinics, Tokyo, Japan) was used to

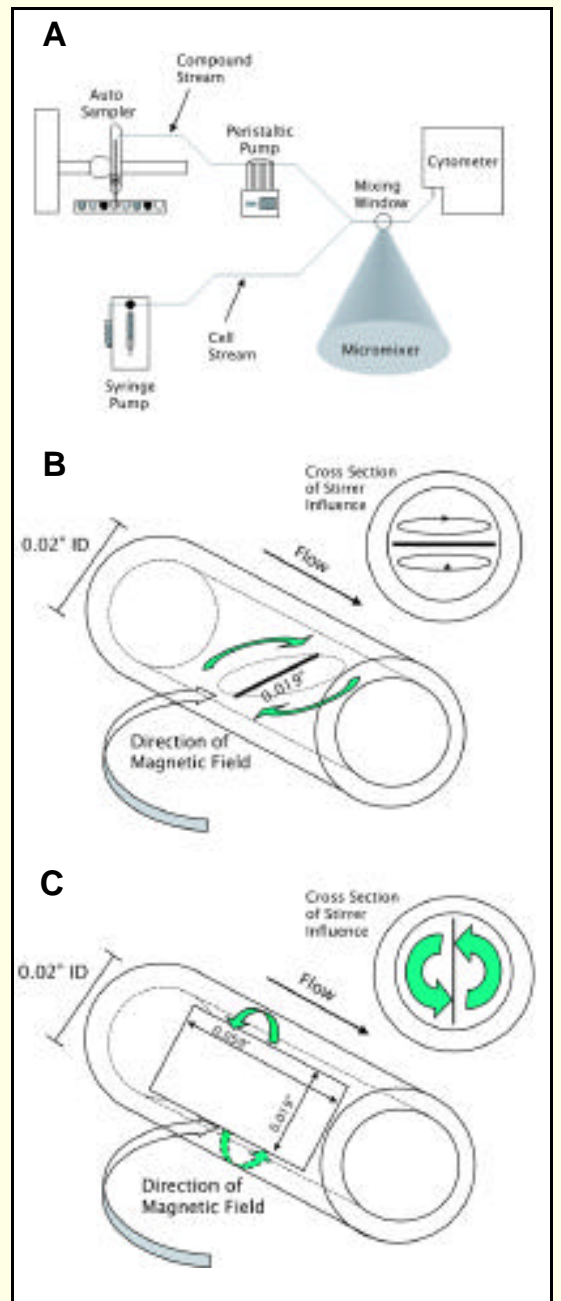

Figure 1. Mixing system and particle resolution. (A) Schematic of the mixing system. The system includes an autosampler with peristaltic tubing connected to one bifurcation of a $\mathrm{Y}$ fitting. The other segment of the $\mathrm{Y}$ is a continuous stream of particles delivered with a syringe. These two streams exit through a common outlet and are mixed before reaching the flow cytometer. The magnetic stirrer is rotating in a plane below the horizontal tubing. (B) An enlarged cross-sectional view of the wire mixer that alters laminar flow due to rotation. (C) The bar mixer was implemented to enhance mixing of the laminar flow from sample and particle streams. The axis of bar rotation is along the length of the tubing. manipulate the in-line mixer and com bine the fluidic streams. Based on the performance of the wires, we cut Metglas ${ }^{\circledR}$ Magnetic Alloy 2714A (AlliedSignal, Morristown, NJ, USA) to create a larger in-line bar (Figure 1C). A magnetic tool was used to insert the bar into the line. It was positioned by a thin rod inserted into the line. The 75$\mu \mathrm{m}, 0.003$-in-thick sheet of alloy was cut into strips of 0.059 in (length) and 0.019 in (width) to accommodate the width of the tubing. Both devices were maintained at approximately $500 \mathrm{rpm}$ that varied in gas or liquid medium.

\section{Bead Assay Materials}

The 6.2- $\mu \mathrm{m}$ diameter streptavidincoated polystyrene beads (Spherotech, Libertyville, IL, USA) were obtained as $0.5 \%(\mathrm{w} / \mathrm{v})$ suspensions according to the manufacturer's data sheet. PI beads and Flow Check fluorescent beads were obtained from Beckman Coulter (Miami, FL, USA). Biotin and fluorescein biotin were purchased from Molecular Probes (Eugene, OR, USA) and used without further purification.

\section{Bead Preparation}

A $200-\mu \mathrm{L}$ suspension of streptavidin-coated beads $\left(3.7 \times 10^{7}\right.$ beads/ $\mathrm{mL}$ ) was diluted to $4 \mathrm{~mL}$ in Tris buffer, (100 mM Tris-HCl, $150 \mathrm{mM} \mathrm{NaCl}$, $0.1 \%$ BSA, $0.02 \%$ sodium azide, $\mathrm{pH}$ 7.5). Thereafter, $1.0 \mu \mathrm{L} 8.7 \times 10^{-6} \mathrm{M}$ fluorescein biotin solution was added to the beads. The sample was agitated for $20 \mathrm{~min}$ and then centrifuged and resuspended in $6.0 \mathrm{~mL}$ buffer. The final bead suspension was composed of approximately $8 \times 10^{5}$ beads $/ \mathrm{mL}$ with a million fluorescein biotin molecules per bead (2). For the unquenching reaction of the beads, biotin was added to the bead preparation.

\section{Cell Culture}

U937 parental cells and cells expressing the $\triangle \mathrm{ST}$ FPR mutant receptor were transfected as previously described (9). The mutant has serines and threonines deleted from the tail, yielding non-desensitizing cells in which the calcium response is longer lived. Plasticware was obtained from VWR Scien- 
tific. Chemicals and reagents were obtained from Sigma (St. Louis, MO, USA), except where otherwise noted. Cells were grown in tissue culture-treated flasks (Corning, Corning, NY, USA) in RPMI 1640 (HyClone, Logan, UT, USA) containing 10\% FBS (HyClone), $2 \mathrm{mM}$ L-glutamine, $10 \mathrm{mM}$ HEPES, with $10 \mathrm{U} / \mathrm{mL}$ penicillin, and $10 \mu \mathrm{g} / \mathrm{mL}$ streptomycin. Cultures were grown at $37^{\circ} \mathrm{C}$ with $5 \% \mathrm{CO}_{2}$ and passaged from subconfluent cultures every 3-4 days by reseeding at $2 \times 10^{5}$ cells $/ \mathrm{mL}$.

\section{$\mathrm{Ca}^{++}$Mobilization Assay}

Cells were collected by centrifugation at $400 \times g$ and resuspended at $1 \times$ $10^{6}$ cells $/ \mathrm{mL}$ in HHB buffer $[0.11 \mathrm{M}$ $\mathrm{NaCl}, 30 \mathrm{mM}$ HEPES, $10 \mathrm{mM} \mathrm{KCl,} 1$ $\mathrm{mM} \mathrm{MgCl}_{2}, 10 \mathrm{mM}$ glucose, $0.1 \%$ (w/v) HSA]. Cells (15-20 mL) were incubated with 20 nM Fluo-4 AM (Molecular Probes) for $45 \mathrm{~min}$ at $37^{\circ} \mathrm{C}$ with gentle rocking. Cells were then washed in $\mathrm{HHB}$ and resuspended at $1 \times 10^{6}$ cells/mL in $\mathrm{HHB}$ containing $1.5 \mathrm{mM}$ EGTA. The mobilization of intracellular $\mathrm{Ca}^{++}$by various concentrations of $\mathrm{N}$-formyl-methionine-leucine-phenylalanine (fMLF; Sigma) was monitored in real time on a FACScan ${ }^{\mathrm{TM}}$ flow cytometer (BD Biosciences, San Jose, CA, USA) as fluorescence intensity (FL1) over time. Cells were gated on forward scatter and side scatter to exclude dead cells and debris.

\section{Receptor Expression Determination}

U937 $\Delta$ ST FPR-expressing cells were harvested as described above, washed, and resuspended in HHB on ice. Equilibrium binding of the agonist fMLF-lysine-fluorescein (fMLFKFITC) was carried out for $45 \mathrm{~min}$ on ice, in the absence and presence of a 1000fold molar excess of fMLF, to determine the level of total and nonspecific binding, respectively. Ligand-stained cells were analyzed for mean fluorescent intensity on a FACScan flow cytometer. The percentage of receptor-expressing cells could then be determined.

\section{Mixing and Delivery of Cells or Par- ticles and Reagents}

The sample handling and mixing were based on a previously described peristaltic system $(5,7)$ for the delivery of reagents from wells in combination with syringe-driven delivery of cells (Figure 1A). Experiments were performed on a FACScan flow cytometer equipped with a 488-nm argon ion laser. Samples from a 96-well plate were delivered to the flow cytometer using a Gilson 215 autosampler (Middleton, WI, USA). A Gilson interface module allowed for multi-threading among an MS-DOS computer, a robotic probe arm, and peristaltic pump. The probe was a $0.305-\mathrm{m}(508 \mu \mathrm{m}$ o.d. $\times$ $254 \mu \mathrm{m}$ i.d.), stainless steel, thinwalled tube (Small Parts). The probe was typically attached to flexible PVC tubing $(254 \mu \mathrm{m}, 0.01$ in i.d.; Spectra Hardware, Westmoreland City, PA, USA), $0.5 \mathrm{~m}$ in length and run through a peristaltic pump. This fluid line was attached to one leg of a "Y"-connector (0.013 in i.d.; Small Parts). The other leg of the Y-connector was met with similar tubing, delivering a continuous stream of reacting cells or beads with a syringe pump (FIAlab Instruments, Belleview, WA, USA). For this particular experiment, the flow cytometer was connected via $20-\mathrm{cm}$ tubing $(510 \mu \mathrm{m}$, 0.02 in i.d.) attached to the third leg of the $\mathrm{Y}$; the in-line microfluidic mixing device was located at this segment. All legs of the Y fitting have an i.d. of 406 $\mu \mathrm{m}$. The triangular volume at the junction of the three legs was estimated to be $0.18 \mu \mathrm{L}$. A specialized fitting minimized sample discontinuity between the narrow i.d. delivery tubing and the flow cytometer intake tube. Here, the inclusion of the syringe-dampened pulsatile motion, thus increasing the need for the in-line micromixer.

In-house $\mathrm{C}^{++}$software (CRFsoft), written in modifiable object-oriented modules with Microsoft ${ }^{\circledR}$ Visual $\mathrm{C}^{++}$ (Visual Studio 6.0, Professional Edition), was used to control the probe motions in three dimensions. The 1-mL syringe system was controlled with integrated FIAlab ${ }^{\mathrm{TM}}$ software (FIAlab Instruments). The speed of the peristaltic pump (Gilson, Minipuls ${ }^{\mathrm{TM}} 3$ ) was manually controlled. In a typical experiment, the peristaltic pump ran at $15 \mathrm{rpm}$, which corresponds to $180-200 \mu \mathrm{L} / \mathrm{min}$, with the sample and bubble size controlled by varying sip time. Sample plugs were removed from wells at sam pling times of $300 \mathrm{~ms} /$ well. The size of the sample plug was also regulated by the initial volumes of sample in a given well. Reactant plugs were made smaller by keeping the initial volume of the well at $50 \mu \mathrm{L}$ compared to the rinse wells containing $300 \mu \mathrm{L}$ buffer. For the sam pling sequence comprising the repetitive delivery of a reactant plug followed by 11 rinses, the resultant stream of plugs consists of an estimated $0.6 \mu \mathrm{L}$ reactant and $0.9 \mu \mathrm{L}$ undiluted buffer. The reactants and buffer plugs subsequently combined with the continuous stream of reacting cells or particles at the Y-junction. The next segment of tubing, termed the mixing window, can be varied in length to vary the mixing reaction time depending on the kinetics of the reaction being observed and the sample flow rate. Here, the joined streams produced a laminar flow that was mixed with the in-line micromixer. The syringe-driven cell or particle stream was suspended before delivery through the periodic refilling of the syringe.

\section{Data Analysis}

CELLQuest $^{\mathrm{TM}}$ software (BD Biosciences) was used to acquire the timeresolved event clusters generated by

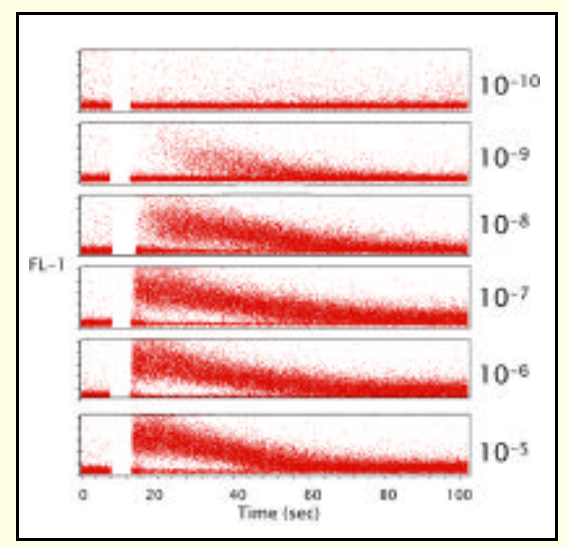

Figure 2. The dose-response for intracellular calcium elevation as mediated by manual mixing and FMLF binding to transfected U937 cells. The data are plotted as dot plots of FL-1 versus time. FL-1 is the cell fluorescence intensity at $520 \mathrm{~nm}$ (bandwidth $30 \mathrm{~nm}$ ). Note that before ligand addition, the cells (each represented by a dot) are clustered near the baseline; following stimulation, the cells respond by the elevation of intracellular calcium and then return to baseline. The final ligand concentrations varied from $1 \times$ $10^{-10}$ to $1 \times 10^{-5} \mathrm{M}$. 
rapid multi-well sampling. Event clusters representing the cell or particle responses to reactants from plates were identified based on variations in fluorescence intensity and analyzed via software (FCSQuery) generated in-house. The software inputs flow cytometry listmode data files stored in FCS 2.0 format and calculates the mean and median fluorescence intensity, as well as the event number and standard deviation of each event cluster. The raw data packet was transferred to Microsoft Excel ${ }^{\circledR}$ for a more detailed analysis.

\section{RESULTS}

\section{Sample Delivery System}

The system (Figure 1A) consisted of a flow cytometer, an autosampler and peristaltic pump for multi-well plates, and a syringe pump for the particles or cells. The syringe was necessary because Fluo-4-loaded U937 cells were spontaneously activated by transit through the peristaltic pump (data not shown). Sample delivery incorporated a peristaltic pump that drew the sample through flexible tubing, which extended from the autosampler to the mixing window. In this segment of tubing, as the probe advanced between wells, air was drawn into the tubing between plugs. The syringe pump supplied the other leg of tubing before the Y fitting. At the Y, the sample, bubble, and wash plugs were brought together with the continuous flow from the cell or particle stream and combined in the mixing window. As described previously (5), the length of the mixing window was determined according to specific reaction kinetics. Two in-line mixers, wire and bar (Figure 1, B and C) were used to disrupt the laminar boundary. Observed under a microscope, the wire mixer rotated in the same direction as the magnetic field, while the bar mixer rotated at right angles to the field, sweeping a path through the laminar boundary.

\section{Bead-Based Assay}

To verify mixing initially, we took advantage of a bead-based assay that would behave similarly to a cell-based receptor-ligand interaction that has been previously described. It is based

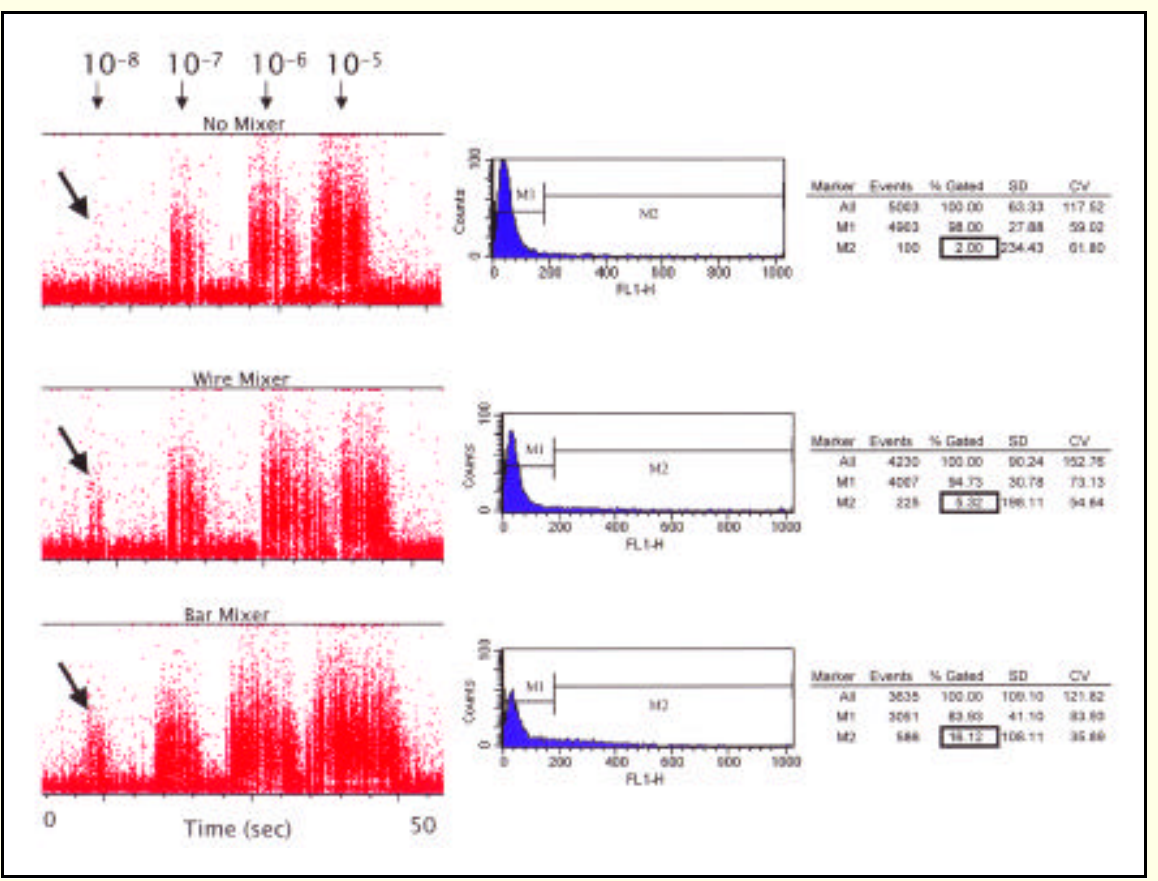

Figure 3. Comparison of the performance of wire and bar micromixers for calcium cell response in Figure 2 using the system shown in Figure 1. Left panels show dose-response sequences for no mixer (top), wire mixer (middle), and bar mixer (bottom). Right panels compare histograms and statistics for cells in responding cell gate M2 as compared to resting cell gate M1. Of note is the appearance of the cells responding to $10^{-8} \mathrm{M}$ peptide. 
on the unquenching by biotin of FITC biotin bound to streptavidin beads. For the beads used here, an 8-fold increase in fluorescence intensity after mixing with excess native biotin could be achieved (data not shown), and it is the relative increase in fluorescence that provides the best indication of mixing.

\section{Cell-Based Assay}

The cell-based assay took advantage of the agonist-dependent calcium response of U937 cells transfected with a mutant FPR. The $\triangle$ ST FPR mutant is slow to desensitize, giving a somewhat prolonged response to ligand binding. Figure 2 shows the dose-response characterization for the calcium elevation at room temperature. The length of the mixing window in subsequent experiments $(8 \pm 2 \mathrm{~s}$ ) was optimized to capture the peak of the response. Typically, $80 \%-90 \%$ of the cells responded with a calcium elevation, and an equilibrium assay for fMLPK-FITC indicated that the percent of responding cells corresponded to the percent of cells that expressed the receptor (data not shown). It is clear from Figure 2 and from previous work that the fraction of responding cells is characteristic of the effective concentration of the ligand.

To test the assay in the sample delivery system, a stream of cells driven by a syringe was allowed to interact with plugs of agonist taken from a 96-well plate by a sample handler via a peristaltic pump. The time course of sam ple delivery from wells containing increasing ligand concentration separated by buffer wells is shown for no mixer, the wire mixer, or the bar mixer. Statistics are shown for the $10^{-8} \mathrm{M}$ ligand concentration at which $2 \%, 5 \%$, or $16 \%$ responding cells are shown for the three mixing conditions (Figure 3 ).

These dose-response data in beads are summarized in Figure 4, A and B, for the wire and the bar mixers. The presence of the wire has little effect until mixing is initiated. In contrast, the bar alone represents a considerable im provement even when not stirring, and when stirring it is nearly as efficient as that obtained by manual mixing, as shown in Figure 2. A similar series of experiments was conducted for the cellbased assay (Figure 5, A and B), show ing the improvement induced by the bar mixer as compared to the wire mixer and by the initiation of stirring. Typically, the introduction of the bar without stirring creates about $30 \%$ of the mixing induced by stirring. Given the factor of two uncertainty in concentration in the delivery system, the stirring bar is identical to the manual mixing.

To verify further the robust nature of the in-line microfluidic mixer, we varied the proportion of non-transfected
U937 cells by increasing the percentage of transfected $\Delta \mathrm{ST}$ from $1 \%$ to $30 \%$ (not shown). Even though as many as $4 \%-5 \%$ of the cells are detected above baseline fluorescence levels in the unstimulated cell population, the response to the stimulus at saturating levels can be detected for $1 \%-30 \%$ of the $\Delta \mathrm{ST}$ cells in either manual or online mixing. The percentages of responding cells corresponded to those percentages of input $\Delta \mathrm{ST}$.

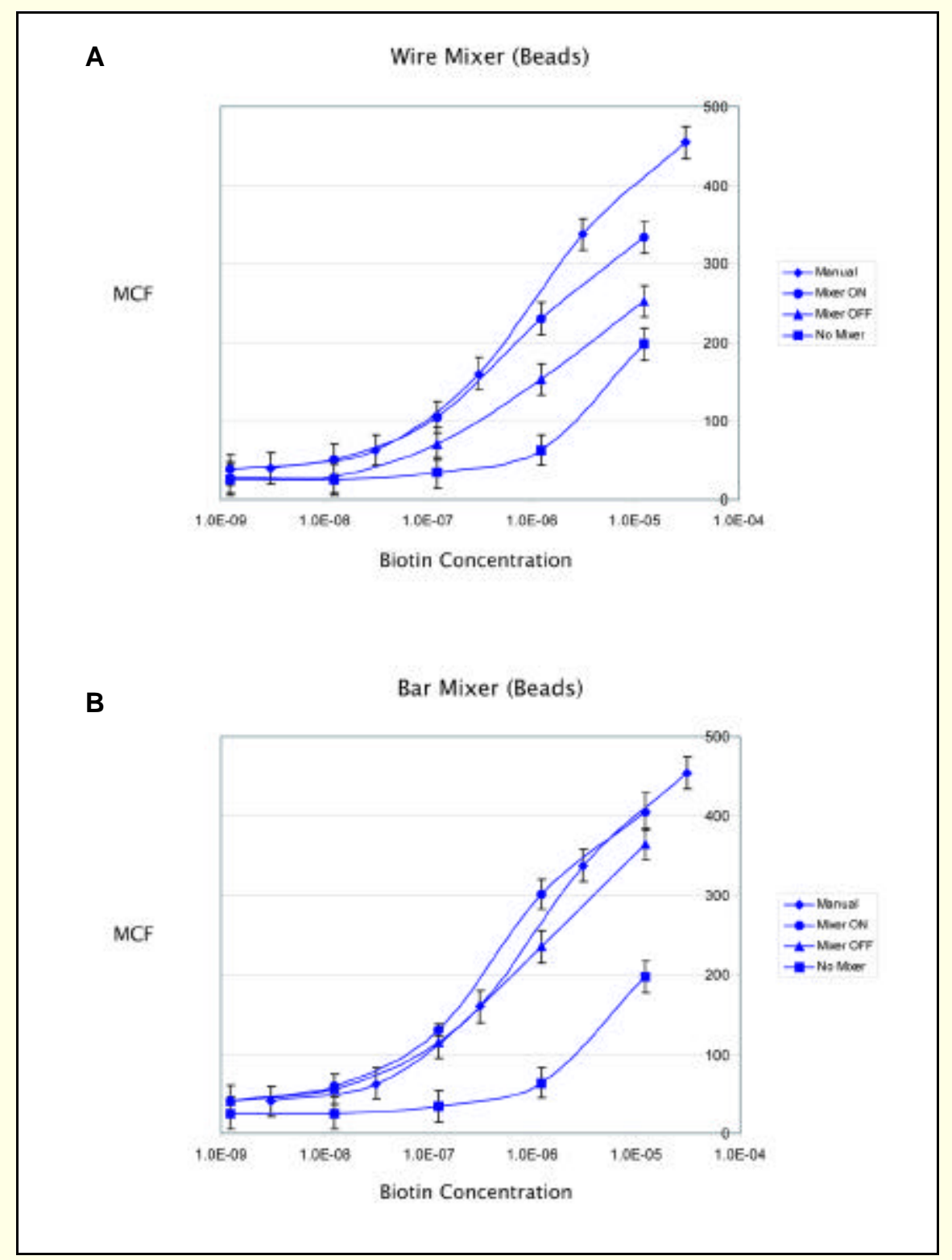

Figure 4. Comparison of mixing devices in bead-based receptor assay. Performance of the wire (A) and bar (B) in-line microfluidic mixing device is plotted as mean channel fluorescence (MCF) versus biotin concentration. The error bars represent the uncertainty due to the distribution of particle responses. In each case, manual responses from Figure 2 are compared to the presence of the mixer with and without stirring or with no mixer. 


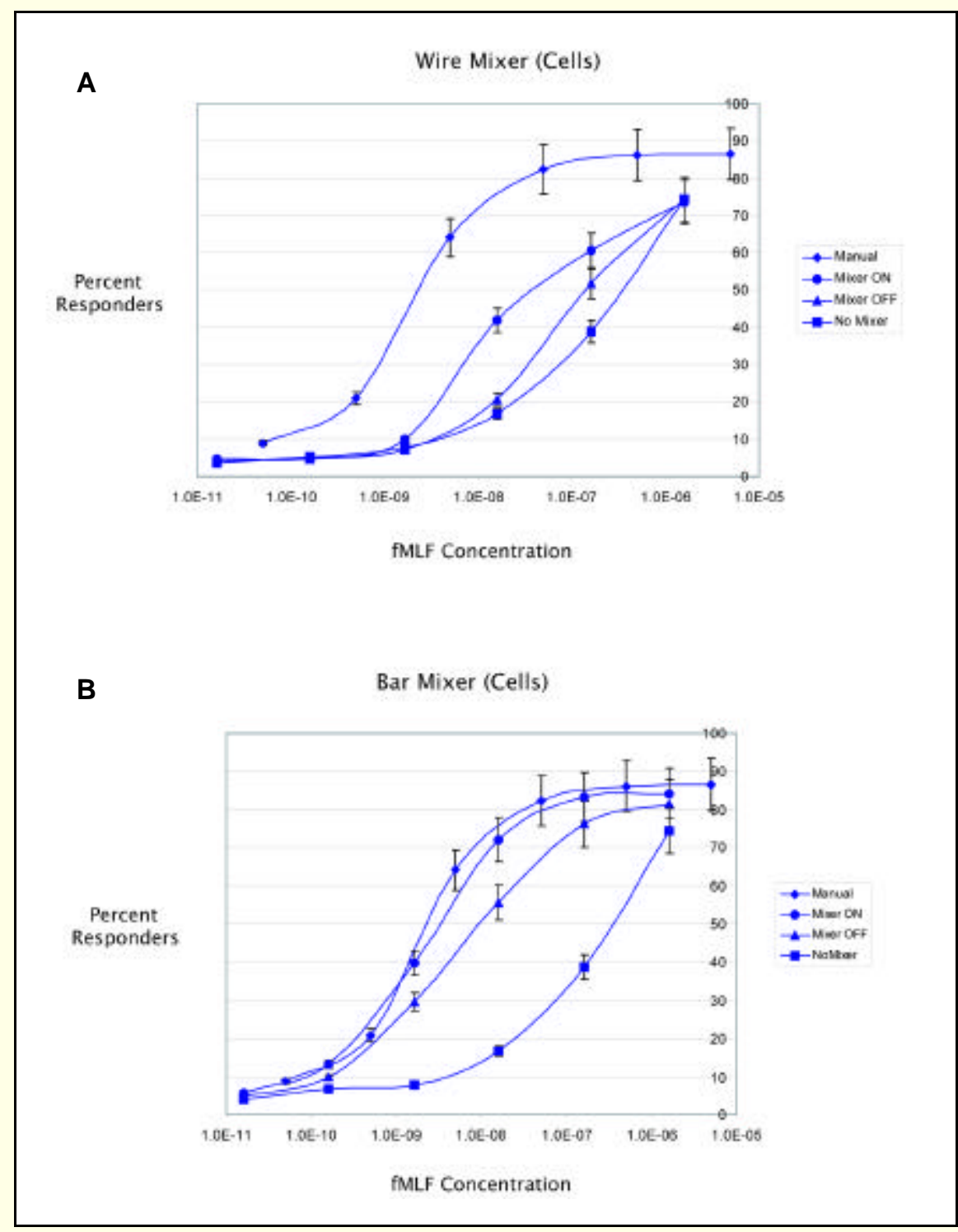

Figure 5. Comparison of mixing devices in cell-based assay. Performance of the wire (A) and bar (B) in-line microfluidic mixing device is plotted as percent responders versus fMLF peptide concentration. The error bars represent the uncertainty due to cell statistics. In each case, manual responses from Figure 2 are compared to the presence of the mixer with and without stirring or with no mixer. The concentrations in the mixing system are estimated by considering the relative volumes of cells, stimulus, and spread of the stimulus over several wells, as discussed elsewhere (5). There is less than a factor of two uncertainty in the effective concentration.

\section{DISCUSSION}

We have previously shown that cell/compound reactions can be mixed online and analyzed in real time using flow cytometry and pulsatile mixing (5). Here, our purpose was to mix small volumes for cell-based analysis of an intracellular calcium response. We could not use the pulsatile mixing approach because the cells became spontaneously activated in transit through the peristaltic pump. The activation of the calcium response was likely caused by flattening of the cells due to com pression of the tubing in the peristaltic pump, a problem that could hinder the real-time analysis of cell responses. In addition, when the pump was used on only one side of the Y, the mixing was inefficient. We modified our previous high-throughput mixing system by combining an automated sample handler having peristaltic pump-driven flu- 
idics with an automated syringe. This system configuration (Figure 1A) allowed for the delivery of a continuous cell or particle stream combined with a non-continuous peristaltic sample stream. With this system, it appears that we achieved homogenous mixing of the two sample lines through analysis in real time via flow cytometry.

To determine the effectiveness of our mixing devices, we illustrated that a bead-based system exposed to a soluble analyte and a receptor agonist cellbased system both produced an increased fluorescence when mixed in a high-throughput system. These fluorescent systems were selected to yield a response when mixed that compared to manual homogenous mixing. The kinetic response characteristics are readily manipulated over the time frame from seconds to minutes through varying the concentration of reactant. In addition, the effective reactant concentrations are in the range of several hundred nanoliters, similar to those desired in drug discovery applications.

Experimental data were analyzed to determine the degree of mixing our inline devices provided. We identified the role of the type of mixing device and how different types of perturbation mix laminar flow streams (Figure 1, B and C). We compared these data with manual batch phase mixing and determined that we have essentially complete mixing under high-throughput conditions (Figures 3-5). It is worth noting that in Figure 2 (manual mixing) and Figure 3 ( $\mathrm{Y}$ and stirrer), similar numbers of cells are detected above the baseline response $(1 \%-3 \%)$ when cells have not been exposed to ligand. This indicates that the microfluidic system with cell delivery with the syringe is not inducing cell activation as the peristaltic pump did. In comparison to the bar mixer, the mixing induced by peristaltic pump delivery of ligand and syringedriven cell delivery through the $\mathrm{Y}$ without any mixer was not effective.

Both cell and bead reactions are concentration-dependent. The calcium responses occur in the time frame of seconds, and the biotin responses occur in the time frame of tens of seconds. Thus, it is necessary to define the length of mixing window necessary to capture a peak response for both bio- logical reactions. By varying the length of tubing post-mixing device, we can define the length of time for a specific reaction to occur.

The throughput of the system is governed by the sampling time of the autosampler (up to $96 \mathrm{wells} / \mathrm{min}$ ) and the number of washes required per sample (3-6 washes; for example, see Figure 3) to eliminate the fluid carryover between samples. This is consistent with 10-20 samples/min (5).

Initially, we used a wire in-line mixing device that did not completely mix the flow streams. The lack of complete mixing was likely due to the minimal perturbation of streamlines above and below the mixer (Figure 1B). Because the behavior of laminar flow and the induction of turbulence are dependent on the dimensions of the fluidic system and the source of perturbation $(1,3)$, we envisioned that improved mixing would result from a microscopic sheet with magnetic properties and an aspect ratio greater than 1 could rotate like a propeller when confined in tubing (Figure 1C). The result was improved microfluidic in-line mixing. The improved mixing may reflect a large mixing surface area and motion orthogonal to the boundary as compared to the wire. It is interesting to note that the presence of the bar alone induced significant mixing, likely as a result of it slicing through the vertical laminar boundaries.

\section{ACKNOWLEDGMENTS}

This work was supported by the National Institutes of Health grant nos. GM60799 and AI48517 to L.A.S. and the State of New Mexico Cigarette Tax. We thank Dr. Hy Tran for the suggestion of MetGlas, Mr. Brett Andrzejewski for his contribution to CRFsoft, and Susan Young for cell preparation. HyperCyt $\mathrm{t}^{\mathrm{TM}}$ is patent pending (trademark filed).

\section{NOTE ADDED IN PROOF}

During the review process, it was pointed out that in-line stirrers have been described in L.H. Lu et al., p. 2830. Proceedings of the Total Micro Analysis Systems (2001) and D.J Beebe et al., p. 453-455. Kluwer Acad- emic Publishers, Dordrecht, edited by J. Michael Ramsey and Albert van den Berg; October 15-21, 2001).

\section{REFERENCES}

1.Beard, D.A. 2001. Taylor dispersion of a solute in a microfluidic channel. J. Appl. Physics 89:4667-4669.

2.Buranda, T., G.M. Jones, J.P. Nolan, J. Kiej, G.P. Lopez, and L.A. Sklar. 1999. Ligand receptor synamics at streptavidin-coated particle surfaces: a flow cytometric and spectofluorimetric study. J. Phys. Chem. 103:3399-3410.

3.Brody, J.P., P. Yager, R.E. Goldstein, and R.H. Austin. 1996. Biotechnology at low Reynolds numbers. Biophys. J. 71:3430-3441.

4.Ethers, S., K. Elgeti, T. Menzel, and G. Weissmeier. 2001. Mixing in the offstream of a microchannel system. Chem. Eng. Processing 39:291-298.

5.Jackson, W.C., F. Kuckuck, B.S. Edwards, A. Mammoli, C.M. Gallegos, G.P. Lopez, T. Buranda, and L.A. Sklar. 2002. Mixing small volumes for continuous high throughput flow cytometry: performance of a mixing $\mathrm{Y}$ and peristaltic sample delivery. Cytometry 47:183-191.

6.Knight, J.B., V. Ashvin, J.P. Brody, and R.H. Austin. 1998. Hydrodynamic focusing on a silicon chip: mixing nanoliters in microseconds. Phys. Rev. Lett. 80:3863-3866.

7.Kuckuck, F., B.S. Edwards, and L.A. Sklar. 2001. High throughput flow cytometry. Cytometry 44:83-90.

8.Monahan, J., A.A. Gewirht, and R.G. Nuzzo. 2001. A method for filling complex polymeric microfluidic devices and arrays. Anal. Chem. 73:3193-3197.

9. Prossnitz, E.R. 1997. Desenitization of Nformylpeptide receptor-mediated activation is dependent upon receptor phosphorylation. J. Biol. Chem. 272:15213-15219.

10.Unger, M.A., H.P. Chou, T. Thorsen, A. Scherer, and S.R. Quake. 2000. Monolithic microfabricated valves and pumps by multilayer soft lithography. Science 288:113-116.

Received 16 October 2001; accepted 12 February 2002.

\author{
Address correspondence to: \\ Dr. Larry A. Sklar \\ Cytometry, Cancer Research Facility \\ University of New Mexico \\ Health Sciences Center \\ Albuquerque, NM 87131, USA \\ e-mail: lsklar@salud.unm.edu
}

